\title{
An Existence Result of Positive Solutions for Fully Second-Order Boundary Value Problems
}

\author{
Yongxiang Li and Yaya Shang \\ Department of Mathematics, Northwest Normal University, Lanzhou 730070, China \\ Correspondence should be addressed to Yongxiang Li; liyx@nwnu.edu.cn
}

Received 28 October 2015; Accepted 7 December 2015

Academic Editor: Kishin Sadarangani

Copyright (C) 2015 Y. Li and Y. Shang. This is an open access article distributed under the Creative Commons Attribution License, which permits unrestricted use, distribution, and reproduction in any medium, provided the original work is properly cited.

An existence result of positive solutions is obtained for the fully second-order boundary value problem $-u^{\prime \prime}(t)=$ $f\left(t, u(t), u^{\prime}(t)\right), t \in[0,1], u(0)=u(1)=0$, where $f:[0,1] \times \mathbb{R}^{+} \times \mathbb{R} \rightarrow \mathbb{R}$ is continuous. The nonlinearity $f(t, x, y)$ may be sign-changing and superlinear growth on $x$ and $y$. Our discussion is based on the method of lower and upper solution.

\section{Introduction and Main Results}

In this paper we discuss the existence of positive solution for second-order boundary value problem (BVP) with fully nonlinear term:

$$
\begin{aligned}
-u^{\prime \prime}(t) & =f\left(t, u(t), u^{\prime}(t)\right), \quad t \in[0,1], \\
u(0) & =u(1)=0,
\end{aligned}
$$

where $f:[0,1] \times \mathbb{R}^{+} \times \mathbb{R} \rightarrow \mathbb{R}$ is continuous, $\mathbb{R}^{+}=[0,+\infty)$. For the special case of BVP (1) that $f$ is nonnegative and does not contain derivative term $u^{\prime}$, namely, the simply secondorder boundary value problem

$$
\begin{aligned}
-u^{\prime \prime}(t) & =f(t, u(t)), \quad t \in[0,1], \\
u(0) & =u(1)=0,
\end{aligned}
$$

the existence of positive solutions has been discussed by many authors; see [1-7]. In these works, the positivity of the corresponding Green function $G(t, s)$ plays an important role. The positivity guarantees that BVP (2) can be converted to a fixed point problem of a cone mapping in $C(I)$, where $I=[0,1]$. Hence, these authors can apply the fixed point theorems of cone mapping to obtain the existence of positive solutions for BVP (2). But their argument methods are not applicable to BVP (1), since these methods cannot deal with the derivative term $u^{\prime}$.
For the more general BVP (1), the existence of solutions and multiple solutions has been discussed by some authors; see [8-11]. In [8-11], the authors have obtained some of the existence results of one solution or multiple solutions by using lower and upper solutions method. But, there are only a few results $[12,13]$ on the existence of positive solutions to the general Dirichlet BVP (1). In [12], Zhang discussed the existence of positive solutions by using Leray-Schauder degree theory and proved that BVP (1) has at least one positive solution if $f(t, x, y)$ is nonnegative and sublinear growth on $x$ and $y$; see [12, Theorem 2.1]. Usually the superlinear problems are more difficult to treat than the sublinear problems. In [13], Agarwal et al. obtained existence results of positive solutions of BVP (1) by using the fixed point index in cones when $f(t, x, y)$ is nonnegative. They allow that $f(t, x, y)$ may be superlinear growth on $x$ rather than $y$.

The purpose of this paper is to obtain existence result of positive solution for BVP (1) under the more general case that $f(t, x, y)$ may be sign-changing and superlinear growth on $x$ and $y$. Our main result is as follows.

Theorem 1. Let $f:[0,1] \times \mathbb{R}^{+} \times \mathbb{R} \rightarrow \mathbb{R}$ be continuous. If $f$ satisfies the conditions,

(F1) there exists a positive constant $\sigma>0$ such that

$$
f(t, x, y) \geq \pi^{2} x, \quad \forall(t, x, y) \in I \times[0, \sigma] \times[-\sigma, \sigma],
$$


(F2) there exist nonnegative constants $a, b$ satisfying $a+\pi b<$ $\pi^{2}$ and a positive constant $C_{0}$ such that

$$
\begin{aligned}
f(t, x, y) \leq a x+b|y|+ & C_{0}, \\
& \forall(t, x, y) \in[0,1] \times \mathbb{R}^{+} \times \mathbb{R},
\end{aligned}
$$

(F3) given any $M>0$, there is a positive continuous function $H_{M}(\rho)$ on $\mathbb{R}^{+}$satisfying

$$
\int_{0}^{+\infty} \frac{\rho d \rho}{H_{M}(\rho)}=+\infty
$$

such that

$$
f(t, x, y) \leq H_{M}(|y|), \quad \forall(t, x, y) \in I \times[0, M] \times \mathbb{R},
$$

then BVP (1) has at least one positive solution.

In Theorem 1, besides that nonlinearity $f$ may be signchanging, condition (F3), a Nagumo-type condition, allows that $f(t, x, y)$ may be superlinear growth on $x$ and $y$ but restricts $f$ on $y$ to quadric growth. See Example 4. This case has not been discussed in $[12,13]$.

The proofs of Theorem 1 are based on the method of lower and upper solution. If a function $v \in C^{2}(I)$ satisfies

$$
\begin{aligned}
-v^{\prime \prime}(t) & \leq f\left(t, v(t), v^{\prime}(t)\right), \quad t \in[0,1], \\
v(0) & \leq 0 \\
v(1) & \leq 0
\end{aligned}
$$

we call it a lower solution of BVP (1). If all of the inequality in (7) is inverse, we call it an upper solution of BVP (1). We will use the following well-known lower and upper solution theorem to prove Theorem 1 in the next section.

Theorem A. Let $f:[0,1] \times \mathbb{R}^{+} \times \mathbb{R} \rightarrow \mathbb{R}$ be continuous, and BVP (1) has a lower solution $v_{0}$ and an upper solution $w_{0}$ with $v_{0} \leq w_{0}$. If the nonlinearity $f$ satisfies the Nagumo-type condition (F3), then BVP (1) has at least one solution between $v_{0}$ and $w_{0}$.

For Theorem A, see [11, Theorem 1].

\section{Proof of the Main Results}

Let $I=[0,1]$. Let $C(I)$ denote the Banach space of all continuous function $u(t)$ on $I$ with norm $\|u\|_{C}=\max _{t \in I}|u(t)|$, and generally for $n \in \mathbb{N}, C^{n}(I)$ denote the space of all $n$ thorder continuously differentiable function on $I$ with the norm $\|u\|_{C^{n}}=\sum_{k=0}^{n}\left\|u^{(k)}\right\|_{C}$. Let $C^{+}(I)$ be the cone of nonnegative functions in $C(I)$. Let $H=L^{2}(I)$ be the usual Hilbert space with the interior product $(u, v)=\int_{0}^{1} u(t) v(t) d t$ and the norm $\|u\|_{2}=\left(\int_{0}^{1}|u(t)|^{2} d t\right)^{1 / 2}$. Let $H^{n}(I)$ be the usual Sobolev space. $u \in H^{n}(I)$ means that $u \in C^{n-1}(I), u^{(n-1)}(t)$ is absolutely continuous on $I$, and $u^{(n)} \in L^{2}(I)$. Let $H_{0}^{1}(I)=\left\{h \in H^{1}(I) \mid\right.$ $u(0)=u(1)=0\}$. Then $H_{0}^{1}(I)$ is a Hilbert space with the norm $\|u\|_{H_{0}^{1}(I)}=\left\|u^{\prime}\right\|_{2}$. It is well-known that every $u \in H_{0}^{1}(I)$ satisfies the Poincaré inequality: $\left\|u^{\prime}\right\|_{2} \geq \pi\|u\|_{2}$.

Given $h \in L^{2}(I)$, we consider the linear boundary value problem (LBVP):

$$
\begin{aligned}
-u^{\prime \prime}(t) & =h(t), \quad t \in[0,1], \\
u(0) & =u(1)=0 .
\end{aligned}
$$

Lemma 2. For every $h \in L^{2}(H), L B V P$ (8) has a unique solution $u:=S h \in H^{2}(I)$. Moreover, the solution operator $S: L^{2}(I) \rightarrow H_{0}^{1}(I)$ is completely continuous and its norm satisfies

$$
\|S\|_{\mathscr{B}\left(L^{2}(I), H_{0}^{1}(I)\right)} \leq \frac{1}{\pi}
$$

where $\|S\|_{\mathscr{B}\left(L^{2}(I), H_{0}^{1}(I)\right)}$ denotes the norm of the bounded linear operator space $\mathscr{B}\left(L^{2}(I), H_{0}^{1}(I)\right)$. When $h \in C(I)$, the solution Sh $\in C^{2}(I)$, and the solution operator $S: C(I) \rightarrow C^{1}(I)$ is completely continuous.

Proof. Let $h \in L^{2}(I)$. By the Green function expression of solution of linear boundary value problem, LBVP (8) has a unique solution expressed by

$$
u(t)=\int_{0}^{1} G(t, s) h(s) d s:=\operatorname{Sh}(t)
$$

where $G(t, s)$ is the corresponding Green function given by

$$
G(t, s)= \begin{cases}t(1-s), & 0 \leq t \leq s \leq 1, \\ s(1-t), & 0 \leq s \leq t \leq 1\end{cases}
$$

By (10), we have

$$
u^{\prime}(t)=\int_{t}^{1}(1-s) h(s) d s-\int_{0}^{t} s h(s) d s, \quad t \in I .
$$

From this and (10), we easily see that the solution operator $S: L^{2}(I) \rightarrow H_{0}^{1}(I)$ is completely continuous. When $h \in C(I)$, the solution $S h \in C^{2}(I)$, and the solution operator $S: C(I) \rightarrow$ $C^{1}(I)$ is completely continuous.

Since sine system $\{\sin k \pi t \mid k \in \mathbb{N}\}$ is a complete orthogonal system of $L^{2}(I)$, every $h \in L^{2}(I)$ can be expressed by the Fourier series expansion

$$
h(t)=\sum_{k=1}^{\infty} h_{k} \sin k \pi t,
$$

where $h_{k}=2 \int_{0}^{1} h(s) \sin k \pi s d s, k=1,2, \ldots$, and the Parseval equality

$$
\|h\|_{2}^{2}=\frac{1}{2} \sum_{k=1}^{\infty}\left|h_{k}\right|^{2}
$$


holds. Let $u=S h$, then $u \in H^{2}(I)$ is the unique solution of LBVP (8), and $u$ and $u^{\prime \prime}$ can be expressed by the Fourier series expansion of the sine system. Since $-u^{\prime \prime}=h$, by the integral formula of Fourier coefficient, we obtain that

$$
u(t)=\sum_{k=1}^{\infty} \frac{h_{k}}{k^{2} \pi^{2}} \sin k \pi t .
$$

On the other hand, since cosine system $\{\cos k \pi t \mid k=$ $0,1,2, \ldots\}$ is another complete orthogonal system of $L^{2}(I)$, every $v \in L^{2}(I)$ can be expressed by the cosine series expansion

$$
v(t)=\frac{a_{0}}{2}+\sum_{k=1}^{\infty} a_{k} \cos k \pi t
$$

where $a_{k}=2 \int_{0}^{1} v(s) \cos k \pi s d s, k=0,1,2, \ldots$. For the above $u=S h$, by the integral formula of the coefficient of cosine series, we obtain the cosine series expansions of $u^{\prime}$ :

$$
u^{\prime}(t)=\sum_{k=1}^{\infty} \frac{h_{k}}{k \pi} \cos k \pi t
$$

Now from (17), (13), and Parseval equality, it follows that

$$
\begin{aligned}
\|S h\|_{H_{0}^{1}(I)}{ }^{2} & =\left\|u^{\prime}\right\|_{2}^{2}=\frac{1}{2} \sum_{k=1}^{\infty}\left|\frac{h_{k}}{k \pi}\right|^{2} \leq \frac{1}{2 \pi^{2}} \sum_{k=1}^{\infty}\left|h_{k}\right|^{2} \\
& =\frac{1}{\pi^{2}}\|h\|_{2}{ }^{2} .
\end{aligned}
$$

This means that $\|S\|_{\mathscr{B}\left(L^{2}(I), H_{0}^{1}(I)\right)} \leq 1 / \pi$, namely (9) holds.

Lemma 3. Let $a, b$ be nonnegative constants and satisfy $a+$ $\pi b<\pi^{2}$; then for every nonzero $h \in C^{+}(I)$, the boundary value problem

$$
\begin{aligned}
-u^{\prime \prime}(t) & =a u(t)+b\left|u^{\prime}(t)\right|+h(t), \quad t \in[0,1], \\
u(0) & =u(1)=0
\end{aligned}
$$

has a unique positive solution $w \in C^{2}(I)$.

Proof. We define a mapping $F: H_{0}^{1}(I) \rightarrow L^{2}(I)$ by

$$
F(u)(t):=a u(t)+b\left|u^{\prime}(t)\right|+h(t),
$$

$$
u \in H_{0}^{1}(I), t \in I .
$$

By Lemma 2, the composite mapping $A=S \cdot F: H_{0}^{1}(I) \rightarrow$ $H_{0}^{1}(I)$ is well defined and the solution of BVP (19) is equivalent to the fixed point of $A$. For every $u_{1}, u_{2} \in H_{0}^{1}(I)$, by definition (20) and the Poincáre inequality, we have

$$
\begin{aligned}
\left\|F\left(u_{2}\right)-F\left(u_{1}\right)\right\|_{2} & \leq a\left\|u_{2}-u_{1}\right\|_{2}+b\left\|u_{2}^{\prime}-u_{1}^{\prime}\right\|_{2} \\
& \leq\left(\frac{a}{\pi}+b\right)\left\|u_{2}^{\prime}-u_{1}^{\prime}\right\|_{2} .
\end{aligned}
$$

Hence by (9),

$$
\begin{aligned}
\| & A\left(u_{2}\right)-A\left(u_{1}\right)\left\|_{H_{0}^{1}(I)}=\right\| S\left(F\left(u_{2}\right)-F\left(u_{1}\right)\right) \|_{H_{0}^{1}(I)} \\
& \leq\|S\|_{\mathscr{B}\left(L^{2}(I), H_{0}^{1}(I)\right)} \cdot\left\|F\left(u_{2}\right)-F\left(u_{1}\right)\right\|_{2} \\
& \leq\left(\frac{a}{\pi^{2}}+\frac{b}{\pi}\right)\left\|u_{2}^{\prime}-u_{1}^{\prime}\right\|_{2} \\
& =\left(\frac{a}{\pi^{2}}+\frac{b}{\pi}\right)\left\|u_{2}-u_{1}\right\|_{H_{0}^{1}(I)} .
\end{aligned}
$$

Since $\left(a / \pi^{2}+b / \pi\right)<1$, this means that $A: H_{0}^{1}(I) \rightarrow H_{0}^{1}(I)$ is a contraction mapping. Hence, $A$ has a unique fixed point $w \in H_{0}^{1}(I)$. By Lemma $2, w=S(F(w)) \in H^{2}(I) \subset C^{1}(I)$. By (20), $F(w) \in C(I)$, and hence $w=S(F(w)) \in C^{2}(I)$ is unique solution of BVP (19).

Set $h_{0}=b|w(t)|+h(t)$. Then $h_{0} \in C^{+}(I)$ is nonzero and $w$ is a solution of the linear boundary problem

$$
\begin{aligned}
-u^{\prime \prime}(t)-a u(t) & =h_{0}(t), \quad t \in[0,1], \\
u(0) & =u(1)=0 .
\end{aligned}
$$

By the Green function expression of solution of linear boundary value problem, $w$ is expressed by

$$
w(t)=\int_{0}^{1} G_{a}(t, s) h_{0}(t) d s, \quad t \in I,
$$

where $G_{a}(t, s)$ is the corresponding Green function. By the assumption of Lemma 3, $0 \leq a<\pi^{2}$. If $a=0, G_{a}(t, s)$ is given by (11), and if $0<a<\pi^{2}, G(t, s)$ is given by

$$
G_{a}(t, s)= \begin{cases}\frac{\sin \sqrt{a} t \cdot \sin \sqrt{a}(1-s)}{\sqrt{a} \sin \sqrt{a}}, & 0 \leq t \leq s \leq 1, \\ \frac{\sin \sqrt{a} s \cdot \sin \sqrt{a}(1-t)}{\sqrt{a} \sin \sqrt{a}}, & 0 \leq s \leq t \leq 1 .\end{cases}
$$

By (25) or (11), $G_{a}(t, s)>0$ for every $t, s \in(0,1)$. Hence from (24) it follows that $w(t)>0$ for every $t \in(0,1)$. This means that $w$ is a unique positive solution of BVP (19).

Proof of Theorem 1. We use Theorem A to prove Theorem 1. Choose a positive constant $\varepsilon \in\left(0, \min \left\{\sigma / \pi,\left(\pi^{2}-a\right) / C_{0}\right\}\right)$ and let $v_{0}=\varepsilon \sin \pi t$, where $\sigma, a$, and $C_{0}$ are the constants in Assumptions (F1) and (F2). Since

$$
\begin{aligned}
0 & \leq v_{0}(t) \leq \varepsilon<\sigma, \\
\left|v_{0}^{\prime}(t)\right| & =|\varepsilon \pi \cos \pi t|<\sigma,
\end{aligned}
$$

$t \in I$.

By Assumption (F1), we have

$$
f\left(t, v_{0}(t), v_{0}^{\prime}(t)\right) \geq \pi^{2} v_{0}(t)=-v_{0}^{\prime \prime}(t), \quad t \in I .
$$

Hence $v_{0}$ is a positive lower solution of BVP (1). 
On the other hand, by Lemma 3, the following boundary value problem

$$
\begin{aligned}
-u^{\prime \prime}(t) & =a u(t)+b\left|u^{\prime}(t)\right|+C_{0}, \quad t \in[0,1], \\
u(0) & =u(1)=0
\end{aligned}
$$

has a unique positive solution $w_{0} \in C^{2}(I)$. By Assumption (F2), we have

$$
\begin{aligned}
-w_{0}^{\prime \prime}(t) & =a w_{0}(t)+b\left|w_{0}^{\prime}(t)\right|+C_{0} \\
& \leq f\left(t, w_{0}(t), w_{0}^{\prime}(t)\right), \quad t \in[0,1],
\end{aligned}
$$

and hence $w_{0}$ is a positive upper solution of BVP (1).

Next we show that $v_{0} \leq w_{0}$. Set $u_{0}=w_{0}-v_{0}$; then by the definitions of $v_{0}$ and $w_{0}$, we have

$$
\begin{aligned}
-u_{0}^{\prime \prime}(t) & =-w_{0}^{\prime \prime}(t)+v_{0}^{\prime \prime}(t) \\
& =a w_{0}(t)+b\left|w_{0}^{\prime}(t)\right|+C_{0}-\pi^{2} v_{0}(t) \\
& =a u_{0}(t)+b\left|w_{0}^{\prime}(t)\right|+C_{0}-\left(\pi^{2}-a\right) v_{0}(t) \\
& \geq a u_{0}(t)+C_{0}-\left(\pi^{2}-a\right) v_{0}(t) \\
& =a u_{0}(t)+C_{0}-\varepsilon\left(\pi^{2}-a\right) \sin \pi t \\
& \geq a u_{0}(t)+C_{0}-\varepsilon\left(\pi^{2}-a\right) \geq a u_{0}(t),
\end{aligned}
$$

$t \in I$.

Let $h_{0}=-u_{0}^{\prime \prime}(t)-a u_{0}(t)$. Then by (30), $h_{0} \in C^{+}(I)$ and $u_{0}$ is a solution of linear boundary value problem (23). Hence $u_{0}$ can be expressed by

$$
u_{0}(t)=\int_{0}^{1} G_{a}(t, s) h_{0}(s) d s, \quad t \in I .
$$

By the positivity of the Green function $G_{a}(t, s), u_{0}(t) \geq 0$ for every $t \in I$. This means that $v_{0} \leq w_{0}$. Hence, by Theorem A $\operatorname{BVP}(1)$ has a solution $u$ between $v_{0}$ and $w_{0}$, which is a positive solution.

Example 4. Consider the following superlinear second-order boundary value problem:

$$
\begin{aligned}
-u^{\prime \prime}(t) & =a \sqrt{|u(t)|}+b\left|u^{\prime}(t)\right|-c u^{2}(t)-d u^{\prime 2}(t), \\
t \in[0,1], & t \\
u(0) & =u(1)=0,
\end{aligned}
$$

where $a, b, c$, and $d$ are positive constants. Clearly, $u_{0} \equiv 0$ is a trivial solution of BVP (32). We show that BVP (32) has an explicit positive solution when $0<b<\pi$. Corresponding to BVP (1),

$$
\begin{aligned}
f(t, x, y)=a \sqrt{|x|}+b|y|-c x^{2}-d y^{2} & \\
(t, x, y) & \in I \times \mathbb{R}^{+} \times \mathbb{R} .
\end{aligned}
$$

We easily see that $f(t, x, y)$ satisfies Condition (F3). Choosing

$$
\sigma=\min \left\{\frac{a^{2}}{4 \pi^{4}},\left(\frac{a}{2 c}\right)^{2 / 3}, \frac{b}{2 d}\right\},
$$

for every $(t, x, y) \in I \times[0, \sigma] \times[-\sigma, \sigma]$, by (33) we have

$$
\begin{aligned}
f(t, x, y) & =a \sqrt{|x|}\left(1-\frac{c}{a} \sqrt{|x|^{3}}\right)+b|y|\left(1-\frac{d}{b}|y|\right) \\
& \geq \frac{a}{2} \sqrt{|x|} \geq \pi^{2} x .
\end{aligned}
$$

Hence $f(t, x, y)$ satisfies Condition (F1). Letting $0<b<\pi$, we show that $f(t, x, y)$ satisfies Condition (F2). Choose a constant $a_{1}>0$ such that $a_{1}+\pi b<\pi^{2}$. Then for every $(t, x, y) \in I \times \mathbb{R}^{+} \times \mathbb{R}$, by (33) we have

$$
\begin{aligned}
f(t, x, y) & \leq a \sqrt{|x|}+b|y|=2 \cdot \frac{a}{2 \sqrt{a_{1}}} \cdot \sqrt{a_{1} x}+b|y| \\
& \leq a_{1} x+b|y|+\frac{a^{2}}{4 a_{1}} .
\end{aligned}
$$

This means that $f(t, x, y)$ satisfies Condition (F2) for $a_{1}, b$, and $C_{0}=a^{2} / 4 a_{1}$. Hence by Theorem 1 , BVP (32) has a positive solution when $0<b<\pi$ besides the trivial zero solution.

\section{Conflict of Interests}

The authors declare that there is no conflict of interests regarding the publication of this paper.

\section{Acknowledgments}

This research was supported by NNSFs of China (11261053, 11361055) and the NFS of Gansu Province (1208RJZA129).

\section{References}

[1] H. Wang, "On the existence of positive solutions for semilinear elliptic equations in the annulus," Journal of Differential Equations, vol. 109, no. 1, pp. 1-7, 1994.

[2] L. H. Erbe and H. Wang, "On the existence of positive solutions of ordinary differential equations," Proceedings of the American Mathematical Society, vol. 120, no. 3, pp. 743-748, 1994.

[3] L. H. Erbe, S. C. Hu, and H. Wang, "Multiple positive solutions of some boundary value problems," Journal of Mathematical Analysis and Applications, vol. 184, no. 3, pp. 640-648, 1994.

[4] J. Henderson and H. Wang, "Positive solutions for nonlinear eigenvalue problems," Journal of Mathematical Analysis and Applications, vol. 208, no. 1, pp. 252-259, 1997.

[5] Z. Liu and F. Li, "Multiple positive solutions of nonlinear two-point boundary value problems," Journal of Mathematical Analysis and Applications, vol. 203, no. 3, pp. 610-625, 1996.

[6] F. Li and Y. Zhang, "Multiple symmetric nonnegative solutions of second-order ordinary differential equations," Applied Mathematics Letters, vol. 17, no. 3, pp. 261-267, 2004. 
[7] Y. Li, "On the existence and nonexistence of positive solutions for nonlinear Sturm-Liouville boundary value problems," Journal of Mathematical Analysis and Applications, vol. 304, no. 1, pp. 74-86, 2005.

[8] H. B. Thompson, "Second order ordinary differential equations with fully nonlinear two-point boundary conditions. I," Pacific Journal of Mathematics, vol. 172, no. 1, pp. 255-277, 1996.

[9] I. Rachůnková, "Upper and lower solutions and topological degree," Journal of Mathematical Analysis and Applications, vol. 234, no. 1, pp. 311-327, 1999.

[10] I. Rachůnková, "Upper and lower solutions and multiplicity results," Journal of Mathematical Analysis and Applications, vol. 246, no. 2, pp. 446-464, 2000.

[11] J. Henderson and H. B. Thompson, "Existence of multiple solutions for second order boundary value problems," Journal of Differential Equations, vol. 166, no. 2, pp. 443-454, 2000.

[12] G. Zhang, "Positive solutions of two-point boundary value problems for second-order differential equations with the nonlinearity dependent on the derivative," Nonlinear Analysis, vol. 69, no. 1, pp. 222-229, 2008.

[13] R. P. Agarwal, D. O’Regan, and B. Q. Yan, "Multiple positive solutions of singular Dirichlet second-order boundary-value problems with derivative dependence," Journal of Dynamical and Control Systems, vol. 15, no. 1, pp. 1-26, 2009. 


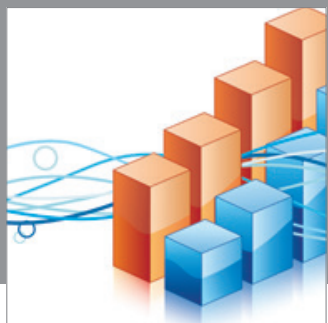

Advances in

Operations Research

mansans

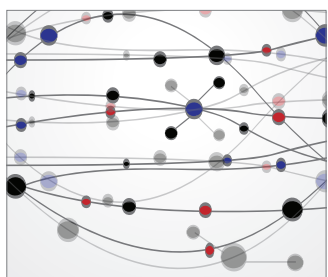

The Scientific World Journal
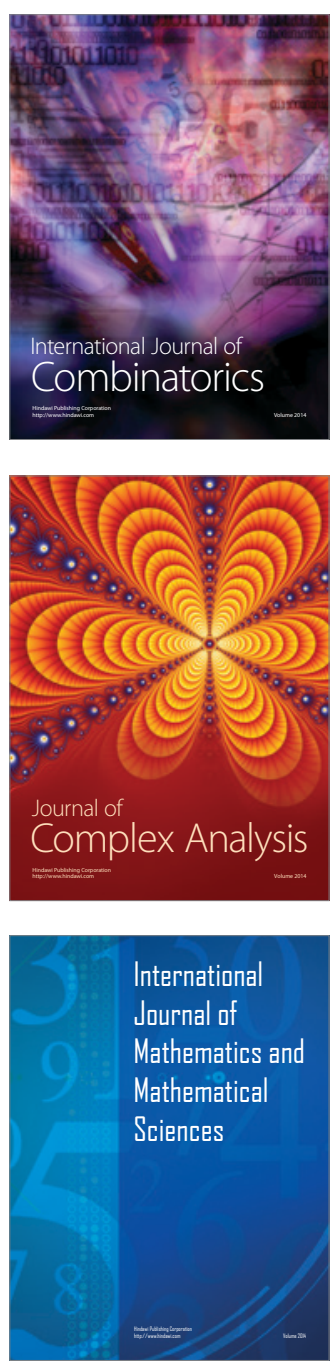
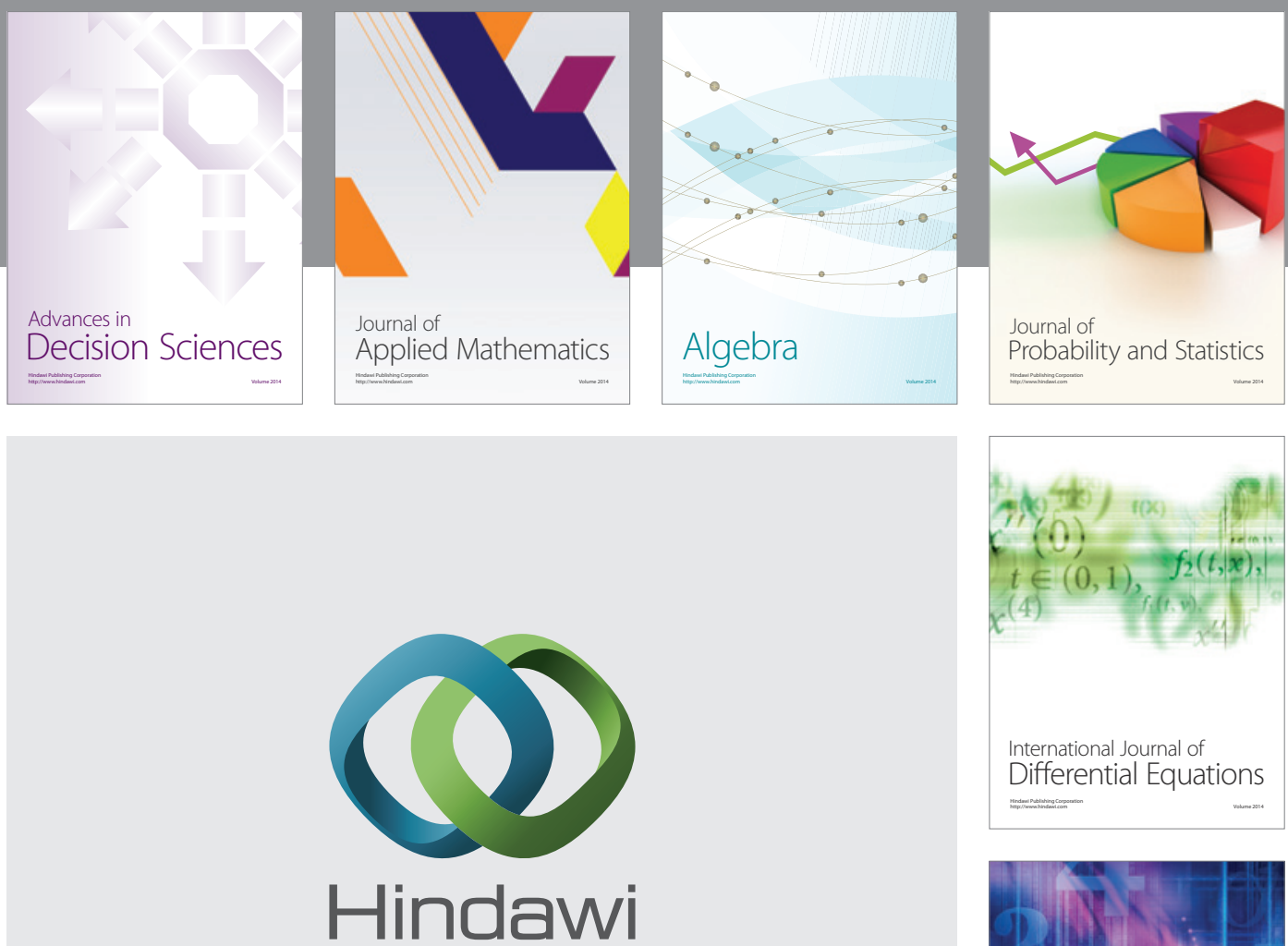

Submit your manuscripts at http://www.hindawi.com
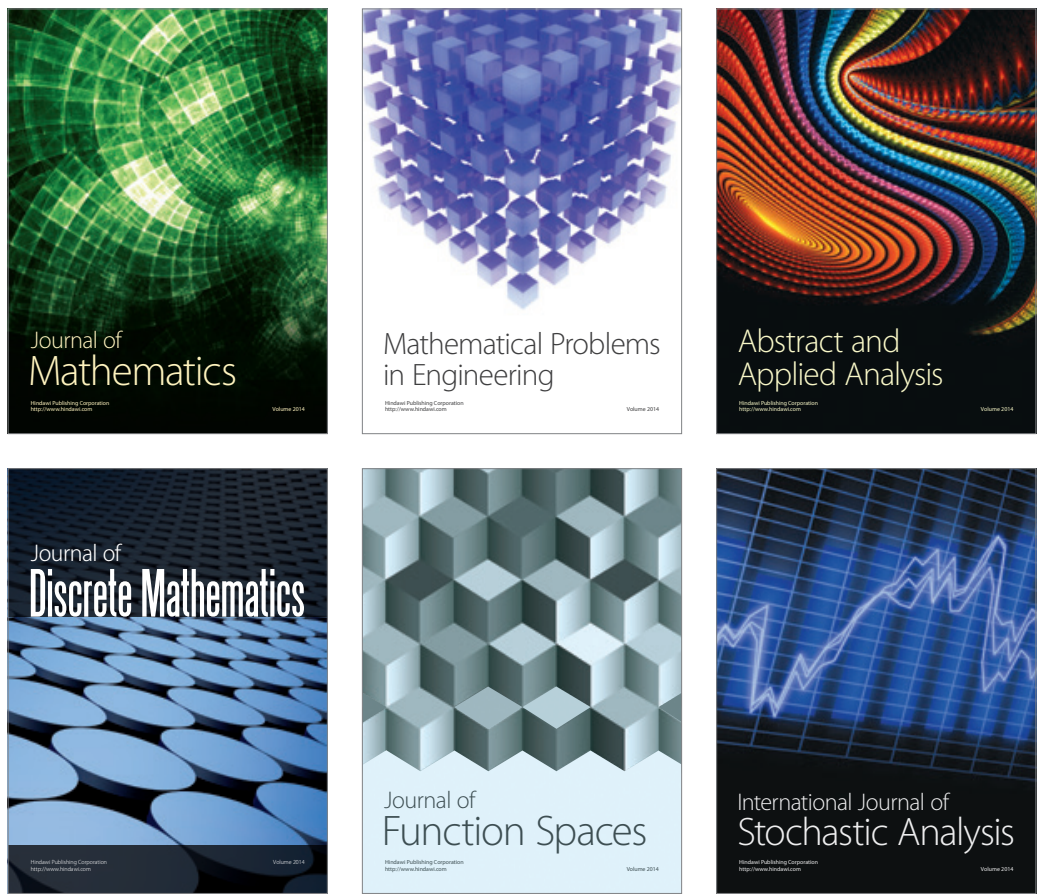

Journal of

Function Spaces

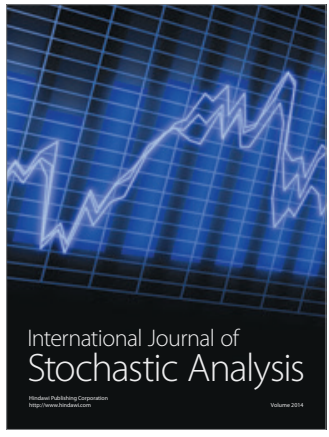

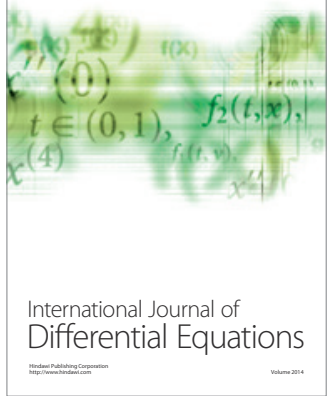
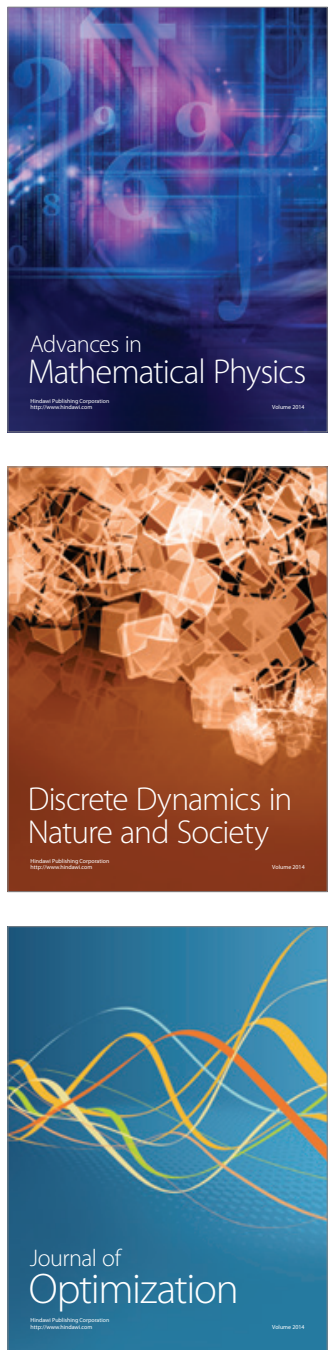